\title{
ANÁLISE DA UNIFORMIDADE DE PAINÉIS DE LED IDENTIFICANDO A INFLUÊNCIA DA DISPOSIÇÃO DOS LEDS NA ESTRUTURA DO PAINEL
}

Bianca Kurihara Yoshii ${ }^{1}$; Felipe Cafezeiro Plech²; Valmara Silveira Ponte ${ }^{3}$; Valéria Loureiro da Silva ${ }^{4}$

${ }^{1}$ Senai Cimatec; Salvador/Bahia; bianca.yoshii.246@gmail.com

${ }^{2}$ Senai Cimatec; Salvador/Bahia;

${ }^{3}$ Senai Cimatec; Salvador/Bahia;

${ }^{4}$ Senai Cimatec; Salvador/Bahia;

Resumo: Atualmente, há diferentes formas de utilização de painéis de LEDs, no entanto, é preciso garantir que estes forneçam uma iluminação uniforme e de qualidade. O objetivo deste trabalho foi realizar um estudo comparativo da uniformidade de dois painéis de LED com iluminação lateral (edge-lit). Para isto foi necessário avaliar a influência da disposição dos LEDs na estrutura do painel e a inserção das máscaras redutoras de área de iluminação. Para aferir tal uniformidade, foram capturadas fotos diretas dos painéis e através do software MATLAB realizados mapas de intensidade de cores falsas e cálculos de desvio padrão. Os resultados indicam que o painel iluminado em dois lados opostos tem significativa uniformidade em relação ao painel iluminado nos 4 lados, independente do uso de máscaras.

Palavras-Chave: Uniformidade luminosa, painel de LED, MATLAB.

\section{LED PANEL UNIFORMITY ANALYSIS IDENTIFYING THE INFLUENCE OF LED ARRANGEMENTS ON THE PANEL STRUCTURE}

\begin{abstract}
There are different ways to use LED panels however, it is necessary to ensure that they provide uniform and quality lighting. The aim of this paper is to compare the uniformity of two edge-lit LED panels through the evaluation of the LEDs arrangement on the panel structure and the insertion of masks. The lit panels were photographed and MATLAB software had be used to create intensity color maps and calculate the standard deviation. The results indicate that the panel with opposite sides illumination produces more uniform backlight than the one illuminated on the four sides, independently of the use of mask.
\end{abstract}

Keywords: Light Uniformity, LED panel, MATLAB. 


\section{INTRODUÇÃO}

A alta eficácia luminosa, o baixo consumo, a longa vida útil, a maior resistência mecânica, o fácil controle sobre a intensidade luminosa e a capacidade de emissão de luz branca, são alguns dos fatores que contribuíram para o desenvolvimento da nova geração de LEDs na iluminação em geral [1] e [2].

Os LEDs (diodos emissores de luz) são semicondutores constituídos por dois materiais diferentes que formam uma junção PN, a qual permite o fluxo de corrente em apenas uma direção. Se a junção PN é polarizada diretamente, as lacunas na camada $\mathrm{P}$ e os elétrons da camada $\mathrm{N}$ movem-se em direção à região de depleção (área de transição entre os materiais $P$ e N). Perto desta região, a recombinação de elétrons e lacunas gera energia que é liberada sob a forma de fótons de luz [3] e [4].

Atualmente há uma grande variedade de formas de utilizar os LEDs, no entanto é preciso garantir que elas forneçam uma iluminação uniforme e de qualidade. Painéis com iluminação lateral por LEDs (edge-lit) foram desenvolvidos para iluminação de painéis LCD e são utilizados em vários tipos de painéis diferentes. Se tornaram populares pelo o melhor aproveitamento do fluxo luminoso e a melhor uniformidade da iluminação. Ao projetar o painel o arranjo dos LEDs deve ser feito de forma a garantir o maior aproveitamento desta iluminação. Diferentes configurações de LEDs como linear, circular, quadrangular e esférica podem ser usadas para se obter uma distribuição luminosa mais uniforme, através da otimização do espaçamento entre eles [5], [6] e [7].

O objetivo deste trabalho é realizar um estudo acerca da uniformidade de dois painéis de LED com iluminação lateral, identificando o quanto esta é influenciada pela disposição dos LEDs na estrutura do painel e o quanto colocar máscaras redutoras de área de iluminação pode melhorar esta uniformidade. O teste foi feito com dois painéis distintos, o painel A possuía 8 LEDs em cada um dos seus dois lados iluminados e o painel B possuía 6 LEDs em cada um dos seus 4 lados iluminados. Foram capturadas fotos diretas dos painéis e através do software MATLAB foi feito um mapa de cores falsas para tratamento dos dados através de cálculos de estatística clássica.

\section{METODOLOGIA}

Foi utilizado um sistema de imageamento óptico (Disponível na Figura 1) para capturar a intensidade da iluminação de cada painel de LED. O mesmo era composto pelo sensor DFK AFU420-CCS de 42 MP, pela objetiva da Navitar com $16 \mathrm{~mm}$ de distância focal e por dois painéis de LEDs, respectivamente.

- Painel A possuía 8 LEDs distribuídos em 2 lados opostos, tamanho de $40 \mathrm{~mm}$ x $40 \mathrm{~mm}$;

- Painel B possuía uma estrutura igual ao painel A, porém, eram 6 LEDs em cada um dos seus 4 lados; 
A partir deste sistema buscou-se o alinhamento da câmera com o painel de LED, enquadrando-o no centro da área de captura. Como esses painéis possuem LEDs muito intensos criou-se uma máscara de $27 \mathrm{~mm} \times 20 \mathrm{~mm}$, esta permitirá avaliar o benefício de se cortar a região mais não homogênea próxima aos LEDs. A abertura da íris e o ajuste do foco foram regulados para uma boa definição da imagem e não saturação da mesma. A figura 2.A e figura 2.B retrata os painéis $A$ e $B$ desligados com máscaras.

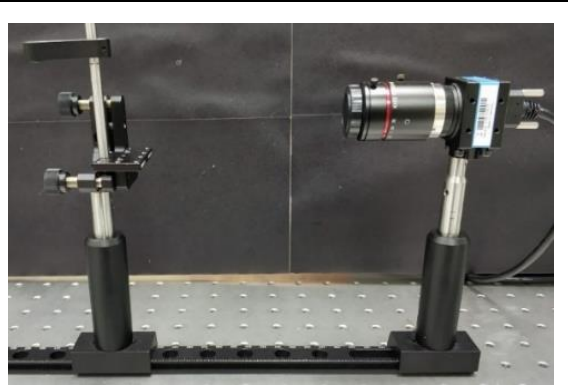

Figura 1: Sistema de imageamento óptico

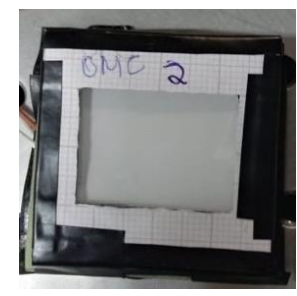

Figura 2.A: Painel A com LEDs em 2 dos seus lados desligado com máscara

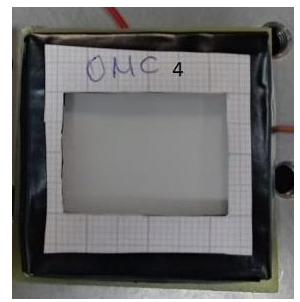

Figura 2.B: Painel B com LEDs em seus 4 lados desligado com máscara

O tratamento dos dados foi feito utilizando o software MATLAB, onde a imagem foi convertida do espaço de cor RGB para tons de cinza, o mesmo gerou um mapa de cor falsa para verificar a uniformidade de luz no painel. Além disso, o script em MATLAB calcula a média e desvio padrão dos valores dos pixels das imagens.

Para determinar os valores de média e desvio padrão, se fez o uso das equações 1 e 2 respectivamente.

$$
\begin{aligned}
& \bar{X}=\frac{1}{N} \sum_{i=1}^{N} X i \\
& \sigma=\sqrt{\frac{\sum_{i=1}^{N}(X i-\bar{X})^{2}}{N}}
\end{aligned}
$$

Para o cálculo do desvio padrão foi necessário transformar os dados da matriz de intensidade de tons cinza de uint8 (dados de imagens armazenados como inteiros de 1 byte) em double. Para análise da uniformidade, foi calculado o desvio padrão total de todos os pixels em tom de cinza assim como o desvio padrão por coluna e por linha. A fórmula std do MATLAB (disponível na equação 2) selecionava cada coluna e percorria a mesma gerando o desvio dela, ao final de todas as colunas plotava-se o gráfico desse desvio padrão em função da posição da coluna. Calculou-se também o desvio padrão para a transposta da matriz de intensidade de tons cinzas. Nesse caso, obtém-se o desvio padrão de cada linha em função da posição da linha, optamos por fazer a média por coluna (ou por linha no caso da transposição) para melhor 


\section{\begin{tabular}{l|l} 
CIRCULAR ECONOMY & ECONOMIA CIRCULAR
\end{tabular}}

representação da uniformidade e otimização de área com valor de desvio padrão desejado.

O script do MATLAB desenvolvido gerava dois mapas de cores falsas, para verificar a uniformidade da intensidade da iluminação de cada painel de LED. Um mapa plotava a matriz de intensidade em escala de tons cinza através da função colormap, e retratava uma barra de intensidade em cores, onde o mínimo e o máximo da intensidade foi determinado a partir da comparação entre as imagens. Já o segundo mapa plotava, através da mesma função, os dados de cada painel normatizados individualmente.

\section{RESULTADOS E DISCUSSÃO}

Após a captura das imagens dos painéis ligados cortou-as para se ter apenas a área do LED (disponível na Figura 3). Nota-se que à medida que o número de lados iluminados com LEDs aumenta, a intensidade luminosa do painel também aumenta.

Figura 3: Imagens capturadas dos painéis ligados

\begin{tabular}{|c|c|}
\hline & \\
\hline $\begin{array}{c}\text { Painel A com LEDs em 2 dos } \\
\text { seus lados ligado com máscara }\end{array}$ & $\begin{array}{c}\text { Painel B com LEDs em seus 4 } \\
\text { lados ligado com máscara }\end{array}$ \\
\hline
\end{tabular}

A figura 4 apresenta os gráficos de cores falsas a partir dos dados de intensidade em escala de cinza. Para este teste a abertura de íris se deu em 5,6, o foco ficou entre os valores de 0,4 - 0,2 e a exposição de 1/12987 segundos.

Figura 4: Mapa de cores falsas com dados de intensidade dos painéis e normalização dos mesmos

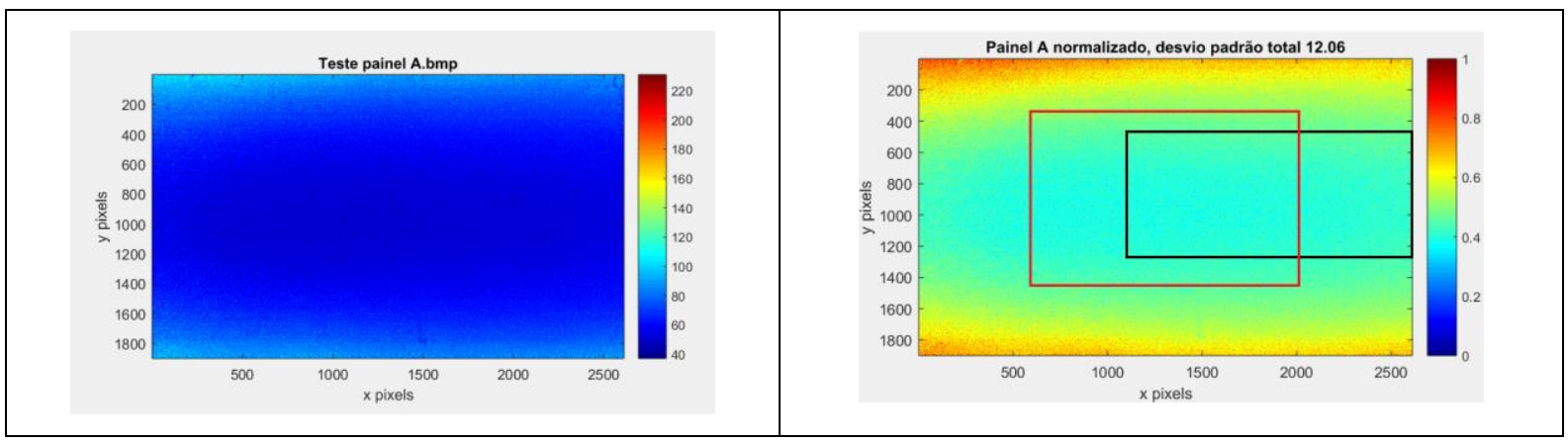




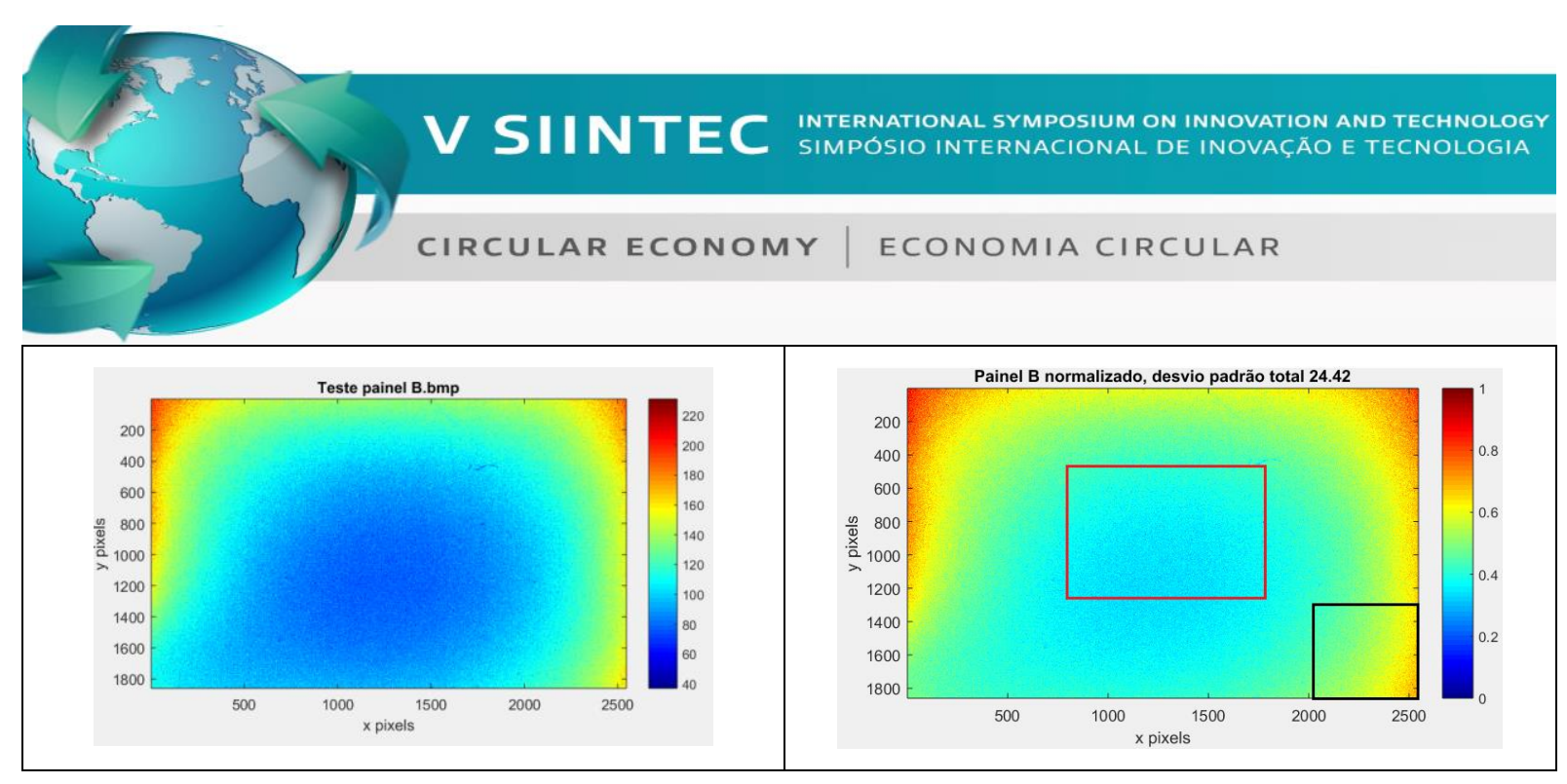

A partir dos mapas da esquerda é possível perceber que a intensidade aumenta à medida que o número de lados iluminado aumenta. Percebe-se que o painel $A$, mesmo sendo mais escuro que o painel $B$, tem a iluminação mais uniforme apresentando metade do desvio padrão do painel B. Os mapas de cores falsas normalizados (mapas da direita) mostram claramente que a área central dos painéis é mais uniforme e pontos quentes aparecem próximo as bordas iluminadas pelos LEDS. Note que a assimetria nos mapas do painel B ocorrem devido à uma má centralização da máscara utilizada.

A Figura 5 apresenta os dados de desvio padrão para as colunas e linhas da matriz dos dados de intensidade em escala de cinza. A partir deste gráfico percebese que 0 desvio padrão aumenta à medida que o número de lados iluminados aumenta. Nota-se que o painel A possui baixa variação de desvio padrão e na sua curva de desvio através da transposta é possível perceber que se cria uma depressão bem no meio do gráfico devido a sua redução de iluminação no meio do painel. Já o painel B possui uma grande variação de desvio padrão, provavelmente decorrente a má uniformidade do mesmo.

Figura 5: Desvio padrão dos dados de intensidade em escala de cinza

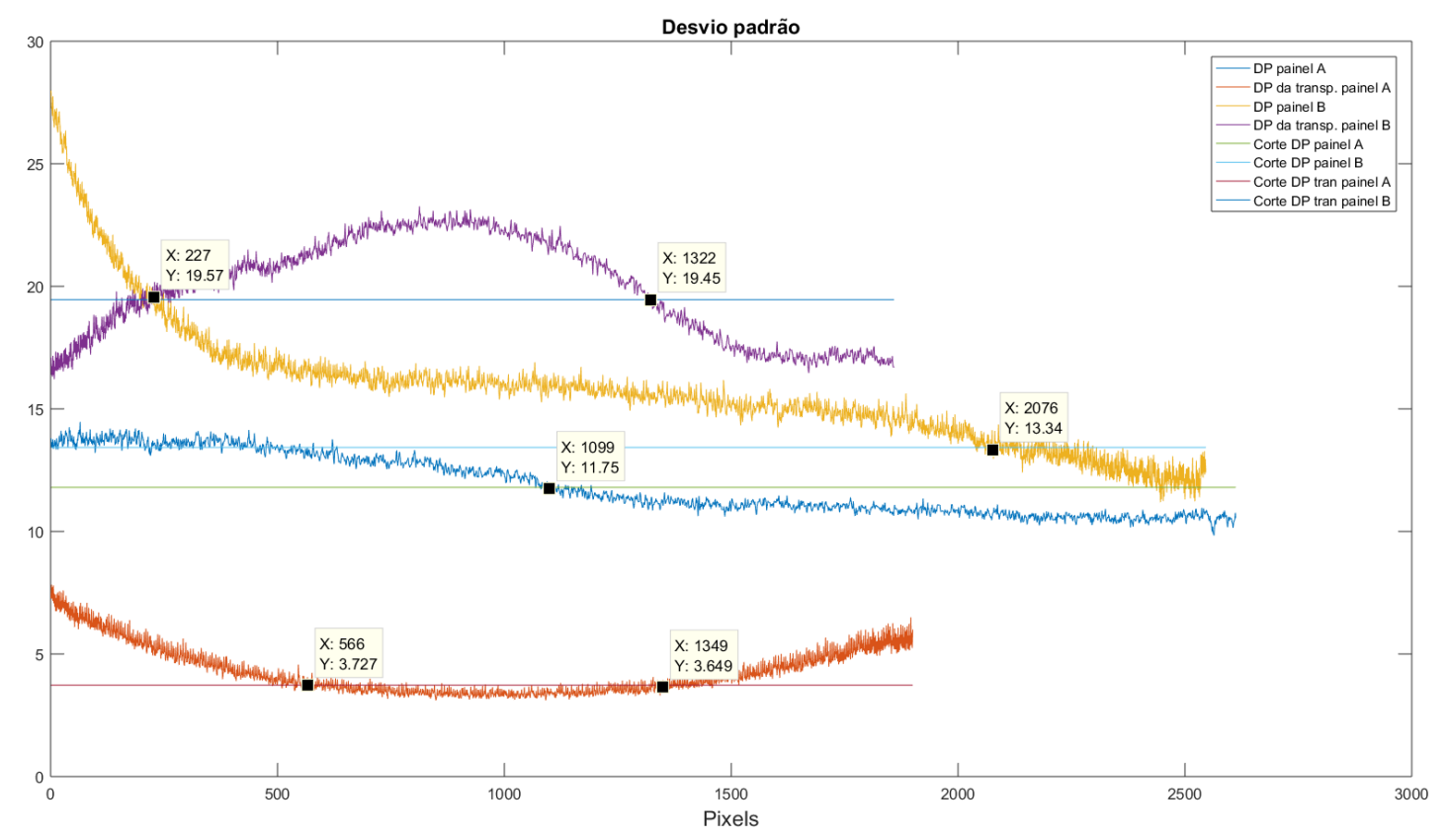


Em algumas aplicações onde a uniformidade é crítica, é possível fazer uso de máscaras para restringir a área útil do painel e melhorar a uniformidade. Para seleção da área ótima que satisfaça um desvio padrão máximo desejado, foi desenvolvida uma metodologia a partir das curvas de desvio padrão das colunas e linha (matriz transposta) mostradas na Figura 5. Esse procedimento é ilustrado abaixo para o caso de desvio padrão máximo desejado de $20 \%$. A figura 6 apresenta os mapas de cores falsas renormalizados para as imagens reduzidas.

Linhas de cortes foram feitas a $20 \%$ dos menores valores de desvio padrão encontrados. Através destes foi possível identificar margens para corte (crop) da matriz, a fim de se fazer uma nova máscara redutora permitindo avaliar a área com o menor desvio padrão. As bordas da máscara obtida para os painéis $A$ e $B$ foram feitos de acordo com os valores de $X$ de cada marcação da Figura 5, resultando nos retângulos de bordas pretas mostrados na Figura 4.

A aplicação de uma máscara otimizada permitiu aumentar significativamente a uniformidade do painel, reduzindo o desvio padrão em 3,5x para 3,35. Já no caso do painel $B$, a redução no desvio padrão após aplicação da máscara é muito menor $(1,6 x)$ e a imagem ainda apresenta não uniformidade significativa, com desvio padrão de 15,60. Essa estratégia de melhoria da uniformidade acarreta numa redução da área útil do painel de $75,73 \%$ e $94,56 \%$ para os painéis $A$ e $B$ respectivamente, e só é possível para aplicações que permitem utilizar um painel maior do que a área útil desejada. Apesar desse procedimento resultar numa área reduzida com melhor uniformidade, a marcação dessas áreas na Figura 4 mostra que elas não foram otimizadas.

Figura 6: Mapa de cores falsas normalizados para as imagens dos painéis $A$ e $B$ reduzidas

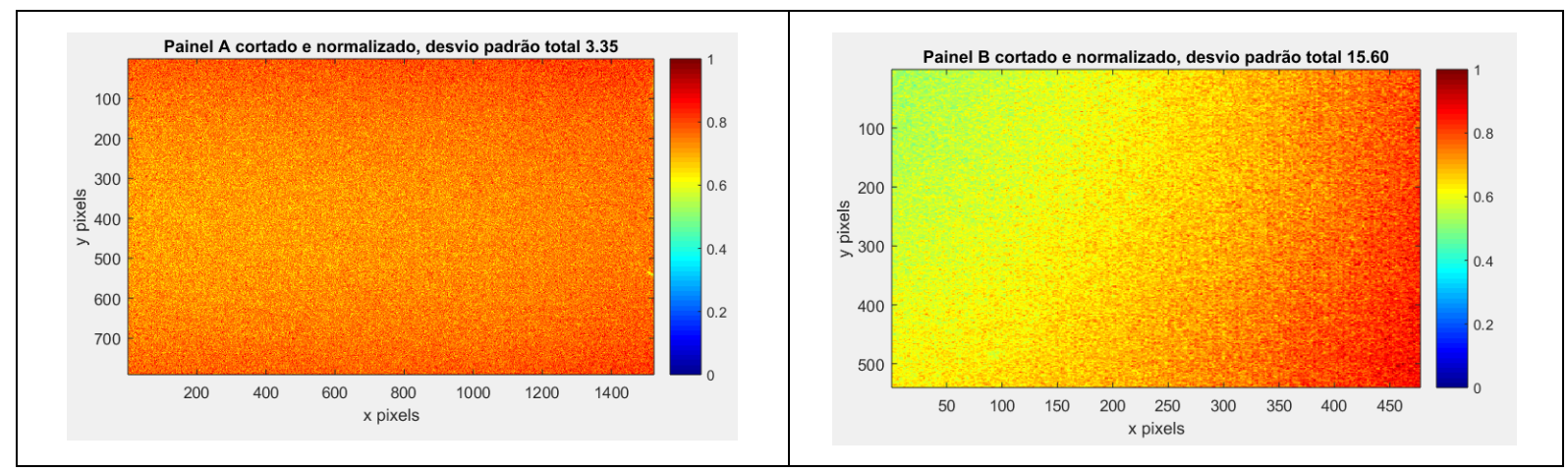

Uma abordagem investigada que pode ser facilmente calculada é escolher as regiões de menor inclinação nas curvas de desvio padrão. A Figura 7 mostra a derivada dessas curvas assim com os pontos para corte indicados na mesma. Através destes cortou-se as curvas do painel A a $20 \%$ dos seus extremos. Já para o painel B cortouse as curvas a $30 \%$ dos seus extremos delimitando a área de corte retratada na Figura 4 pelo retângulo em vermelho e o mapa de cores falsas para esse corte alternativo na Figura 8. Essa nova estratégia mais otimizada, resulta numa redução do desvio padrão total de 2,62x e 3,65x para o painel $A$ e $B$ respectivamente e com uma redução 


\section{\begin{tabular}{l|l} 
CIRCULAR ECONOMY & ECONOMIA CIRCULAR
\end{tabular}}

da área total do painel, de 63,93\% e 83,93\%. Comparado com abordagem de máscara anterior há um aumento modesto no desvio padrão total para o painel $\mathrm{A}$, de 4,60 $\mathrm{x}$ 3,35 , mas um aumento significativo na área útil, $1.204 .693 \times 1.790 .229$. Já para o painel B, o desvio padrão foi reduzido, 6,69 x 15,60 e a área aumentada 257.580 x 761.666, indicando que essa abordagem de corte pelas derivadas é mais otimizada.

Figura 7: Derivada de $1^{\mathrm{a}}$ ordem para o fitting das curvas de desvio padrão dos painéis

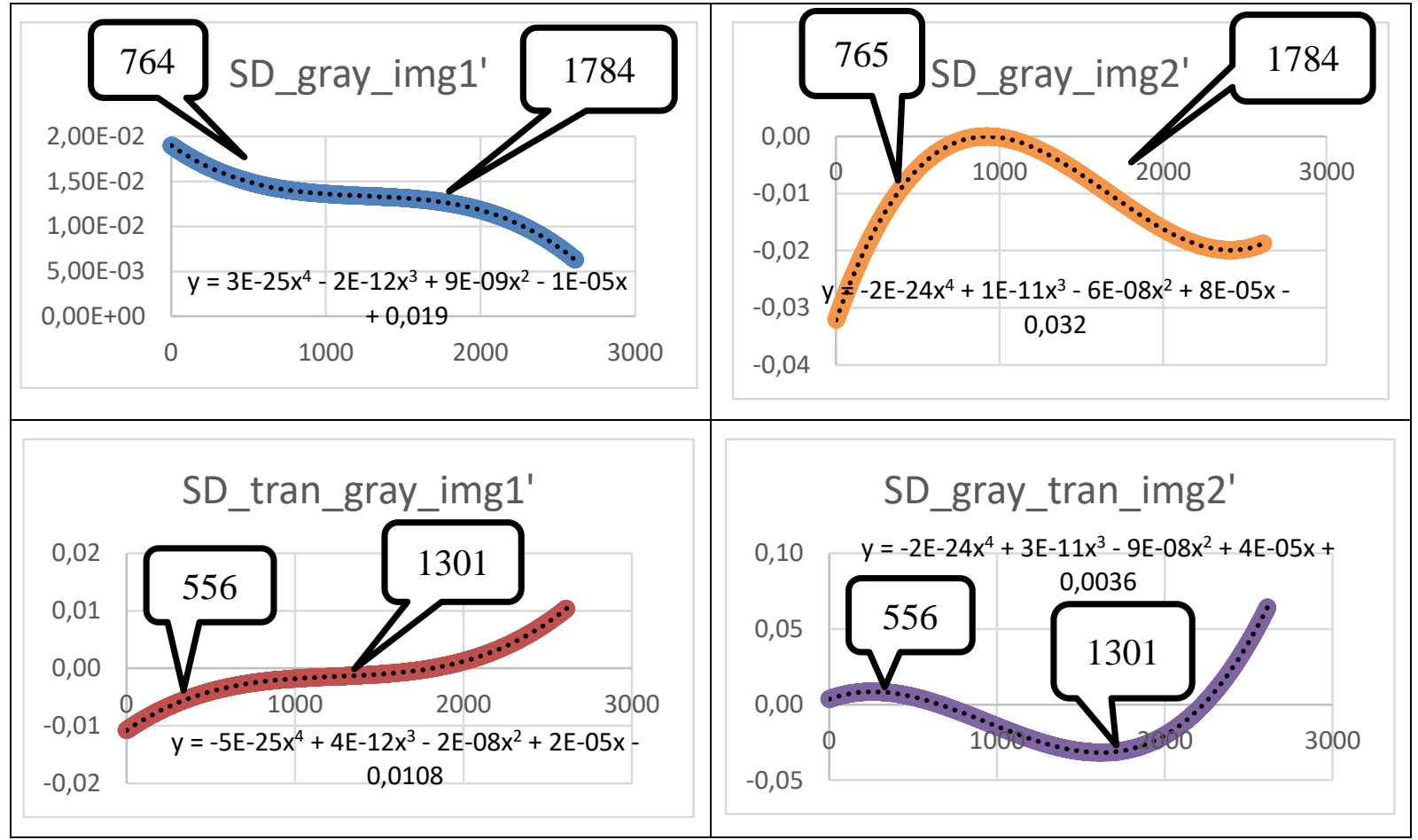

Figura 8: Mapa de cores falsas para o corte alternativo do painel B

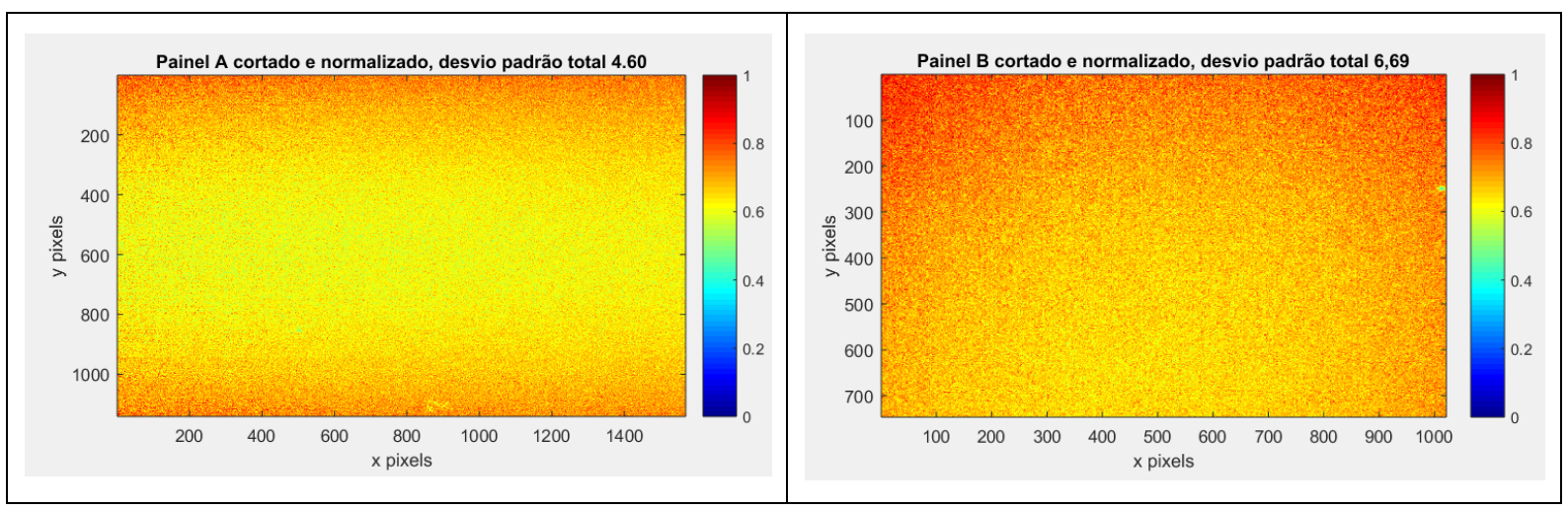




\section{CONCLUSÃO}

A partir dos mapas de cores falsas foi possível caracterizar a uniformidade da intensidade de dois painéis de LEDs retangulares com iluminação lateral (edge-lit). Os resultados mostram que um painel com iluminação em dois lados opostos é significativamente mais uniforme do que um painel iluminado nos 4 lados. Com o uso dos gráficos de desvio padrão foi possível identificar a área mais uniforme dos painéis e o posicionamento ótimo de uma máscara para duas abordagens diferentes. O painel com iluminação em lados opostos novamente apresenta desempenho melhor com relação a uniformidade, com maior redução no desvio padrão da intensidade e maior área útil independente do uso de máscara e abordagem para sua determinação.

\section{Agradecimentos}

Agradecimentos à EMBRAPII, SENAI-CIMATEC e CODEMGE pela bolsa de mestrado de Bianca Kurihara Yoshii.

\section{REFERÊNCIAS}

${ }^{1}$ BALDO, P.; SILVEIRA Jr., L. LED - Aplicações em Luminotécnica. XI INIC Encontro Latino Americano de Iniciação Científica. VII EPG - Encontro Latino Americano de Pós-Graduação. I INIC JR. - Encontro Latino Americano de Iniciação Científica Júnior. UNIVAP. São José dos Campos - SP, Brasil, 18 - 19 outubro, 2007.

2 MAWAKDIYE, A. A Revolução dos LEDs, IPESI Magazine, 157. ed. September/October 2008.

${ }^{3}$ U.S. DEPARTMENT OF ENERGY. Energy Efficiency and Renewable Energy. Thermal Management of White LEDs. November 2009.

${ }^{4}$ BULLOUGH, J. D. Light Emitting Diode Lighting Systems. NLPIP - Lighting Answers. v.7. Issue 3. May, 2003.

${ }^{5}$ DERLOFSKE, J.; Computer modeling of LED light pipe systems for uniform display illumination. Lighting Research Center, Rensselaer Polytechnic Institute, Troy, New York 2001.

${ }^{6}$ SILVA, A.; Reconhecimento automático de defeitos de fabricação em painéis TFT-LCD através de inspeção de imagem. Universidade Federal de Pernambuco Centro de tecnologia e geociências. Programa de pós-graduação em engenharia elétrica. Ano 2016.

${ }^{7}$ WANG, K. et al. New reversing design method for LED uniform illumination. Optics express. 4 Julho de 2011. Vol. 19, número S4. 